\title{
Violencia y gestión escolar: \\ la opinión de directores de escuelas primarias de Colima, México
}

Violence and School Management:

The Opinion of Elementary School Principals

in Colima, Mexico

Violência e gestão escolar:

a opinião de diretores de escolas primarias

de Colima, México

Antonio Gómez-Nashiki* (iD o000-0001-9411-2422

Artículo de investigación

Revista Colombiana de Educación, N. 73. Segundo semestre de 2017, Bogotá, Colombia.

doi: 10.17227/01203916.73rcel7.38:

Para citar este artículo: Gómez, A. (2017). Violencia y gestión escolar : la opinión de directores de escuelas primarias de Colima, México. Revista Colombiana de Educación, (73), 19-40.

\section{(c) $(1) \Theta$}

* Doctor en Pedagogía. Profesor e investigador, Facultad de Pedagogía de la Universidad de Colima. Colima, México. Correo electrónico: gnashiki@ucol.mx 


\section{Resumen}

El artículo analiza la percepción, las implicaciones y las acciones que adoptaron trece directores de escuelas primarias públicas de cinco municipios de Colima para enfrentar distintos problemas de violencia escolar. A partir del análisis institucional, se realizaron entrevistas en profundidad para, posteriormente, contrastar las narrativas de cada caso. Entre los principales hallazgos, los directores señalan que: la gestión se desarrolla bajo mucha presión y demanda soluciones inmediatas ante problemas de distinta índole; e apoyo y la información para atender problemas de violencia escolar son incipientes; consideran poco ágil aplicar los protocolos en caso de violencia; solicitan mayor capacitación en temas como la resolución de conflictos; las acciones acordadas en el Consejo de Participación Social escasamente se cumplen; los padres de familia no apoyan en la solución de los problemas de sus hijos; la violencia genera malestar en la institución y requiere mucha inversión de tiempo para solucionarlo.

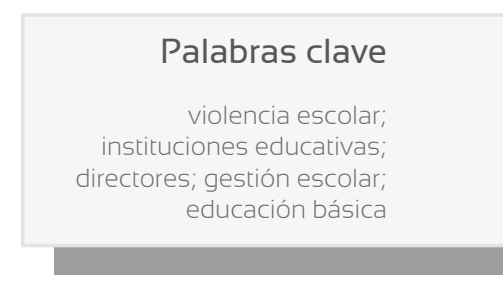

Abstract

This article analyzes the perception, implications and measures taken by thirteen principals from Public Elementary Schools of five municipalities in Colima to deal with different problems of school violence. As part of the institutional analysis, we conducted in-depth interviews in order to compare the narratives in each case. The main findings show that: management is developed under high pressure, and that it demands immediate solutions to different kinds of problems; support and information for facing school violence are incipient; applying the protocols for dealing with school violent events are not expeditious: they demand more training in topics such as conflict resolution; the actions established by the Social Participation Council are rarely followed; parents do not get involved in the solution to children's problems; and violence produces general discomfort in the institution, which requires a lot of time and money to solve it.

\section{Resumo}

O artigo analisa a percepção, as implicações e as ações que adotaram treze diretores de escolas primarias públicas de cinco municípios de Colima para enfrentar diversos problemas de violência escolar. A partir da análise institucional, realizaram-se entrevistas em profundidade para, posteriormente, contrastar as narrativas de cada caso. Entre os principais resultados, os diretores assinalam que: a gestão desenvolve-se sob muita pressão e demanda soluções imediatas ante problemas de diversa índole; o apoio e a informação para atender problemas de violência escolar são incipientes; é pouco ágil aplicar os protocolos em caso de violência; é preciso maior capacitação em temas como a resolução de conflitos; as ações combinadas no Conselho de Participação Social dificilmente são realizadas; os pais de família não ajudam na solução de problemas dos seus filhos; a violência gera mal-estar na instituição e requer muita inversão de tempo para solucioná-la.

\section{Palavras chave}

violência escolar: instituições educativas; gestão escolar; educação

básica 


\section{Estudios sobre directores ${ }^{1}$}

El puesto de director se ha estudiado desde diversas perspectivas; es una posición estratégica que desarrolla muchas actividades y situaciones de manera simultánea todos los días; su labor al frente de la institución es multifacética y difícil de definir: coordina, gestiona, dirige, organiza, negocia, lidera, entre otros aspectos. En relación con la violencia escolar, es un actor central porque le corresponde tomar distintas decisiones, con la finalidad de mejorar la convivencia y evitar la violencia.

Existen varias investigaciones que han abordado las funciones del director en la escuela desde diversos ángulos, por ejemplo: las actividades que realiza cotidianamente (Carriego, 2005; Ezpeleta y Furlan, 1992; Mitzberg, 1983; Pastrana, 1996), el tipo de liderazgo que ejerce (Ball, 1989; Enriquez, 2005; Tesio, 2008); los años de servicio y experiencia en el puesto (García, et al., 2010; Karakose et al., 2014; Kaya, 2015), en relación con su formación ( $\mathrm{Ng}$ y Chan, 2014), la forma en que toma las decisiones (Abdolhamid y Mehdinezhad, 2015), el tipo de interacción que mantiene con el personal de la escuela (Murillo y Hernández-Castilla, 2015); a partir de la variable de género y su estilo de conducir la escuela (Morgade, 2010; Morris et al., 1999); por la capacidad de gestión -tanto administrativa como pedagógica-, que manifiesta ante situaciones críticas (López-Yañez, et al., 2014), o por el sentido y esencia de la tarea que desarrolla (Santos-Guerra, 2015), entre otros aspectos. Estos trabajos han mostrado la complejidad del rol y las diferentes facetas que de manera planeada o imprevista el director debe atender como parte de su quehacer institucional. Sin embargo, son escasos los estudios que abordan las formas de conceptualizar y enfrentar la violencia escolar por parte de este agente.

\section{Objetivos}

El objetivo general de la investigación fue: Analizar los aspectos, consideraciones y decisiones que adoptaron los directores de escuelas primarias públicas de Colima para enfrentar la violencia escolar en su plantel. Los objetivos secundarios fueron: (1) Identificar los conflictos que se presentaron en la institución y el uso de los distintos procedimientos formales e informales para resolverlos, y (2) Caracterizar los aspectos problemáticos de la gestión administrativa y pedagógica que lleva a cabo el director de manera cotidiana.

1 Se habla de directores en general, para referirse a hombres y mujeres que ocupan este puesto de trabajo. 


\section{Preguntas de investigación}

Algunos interrogantes que guiaron la investigación fueron los siguientes: ¿Qué aspectos de las normas, los códigos y valores de la escuela se transmitieron e incorporaron en el proceso de toma de decisiones de los directores y se tradujeron en acciones en torno a los problemas de violencia escolar que les tocó afrontar? ¿Los directores conocen y saben utilizar los protocolos de actuación, así como los procedimientos por seguir para atender los casos de violencia escolar que estableció la Secretaría de Educación Pública? ¿Qué criterios consideraron relevantes y decisivos para resolver este tipo de problemas? ¿Cómo afectó la violencia escolar el desarrollo de la gestión cotidiana?

\section{El supuesto}

De manera cotidiana el director enfrenta y resuelve problemas de distinta índole, conflictos diversos derivados de la interacción cotidiana de los sujetos en la institución. Sin embargo, la violencia escolar, ya sea la que se genera entre alumnos, entre profesores y alumnos, entre alumnos y profesores, entre padres de familia y profesores, o entre padres de familia y alumnos, representa un desafío a su control, así como a los objetivos y fines de la escuela que dirige. Por esta razón, el director utiliza distintos recursos de poder y estrategias a su alcance, tanto formales como informales, para organizar las distintas actividades en la institución. El proceso de gestión incluye la disputa entre los diversos intereses de grupos o sujetos con distintos recursos de poder, que da lugar a amenazas, contraamenazas, coacción, así como los diferentes tipos de violencia: verbal, psicológica, física, sexuada (Gómez-Nashiki, Zurita y López, 2013), que cada uno pone en juego, con la finalidad de lograr que sus fines y objetivos se cumplan, y es en este contexto que el director debe poner en marcha muchos de los recursos a su alcance para poder llevar a cabo los objetivos cuyo cumplimiento considera como prioritario.

\section{Ejes conceptuales}

\section{Institución, dinámica y conflicto}

Las instituciones sociales son formas sociales que definen las condiciones concretas de existencia de las personas, tanto en el plano material como en el concerniente a la generación, propagación y difusión del sistema de normas, valores, prácticas y actitudes compartidas por la sociedad o 
sectores específicos de esta (Fernández, 1995), todo lo cual sirve de marco de referencia a las decisiones y acciones que los individuos realizan en torno a diferentes aspectos que rodean su reproducción (Berguer y Luckmann, 1997; Lourau, 1991).

El carácter permanente de las instituciones no establece de ninguna manera su inmovilidad o estatismo en su interior (Knight, 1992). Por el contrario, al ser producto de la actividad humana se encuentra en continuo movimiento, ya sea para mantener su conservación o para intentar su transformación (Fernández, 2001); movimientos que son generados por la dinámica de la vida colectiva que se genera en su interior.

Las escuelas son arenas de conflicto (Ball, 1989) en donde se dan cita muchas contradicciones entre los individuos. Las funciones establecidas y organizadas dentro de la institución no siempre operan a un nivel de complementariedad y apoyo recíproco, sino que las funciones oficiales y secundarias pueden estar en conflicto, contrarrestando y oponiendo resistencia a las normas o acciones emprendidas o fijadas por otras instituciones. En el mismo espacio institucional coexisten distintos proyectos, así como métodos y procedimientos para alcanzar sus objetivos. Por esta razón, existen distintos niveles de legitimación institucional; el éxito o fracaso de los mecanismos conceptuales de control e imposición de normas e ideologías dependen del poder de aquellos que operan las instituciones, de la autoridad que consiguen detentar y también de la viabilidad que ofrecen a los intereses grupales que las mantienen (Fernández, 1995).

El conflicto que se deriva de la lucha entre los distintos intereses no siempre es violento; sin embargo, en esta investigación se eligieron aquellos testimonios en donde los directores vivenciaron una experiencia de violencia escolar entre los alumnos: física, verbal, sexuada, psicológica.

\section{Liderazgo y estilo institucional}

El liderazgo que representa y asume el director en la institución puede adoptar diversas características, tipos y variantes (Ball, 1989; Meng y Berguer, 2011; Murillo y Hernández-Castilla, 2015), ya no solo centrado en su personalidad, sino también incluyendo al equipo de colaboradores que lo acompañan (Pereira, 2014, p. 103), tomando en consideración el hecho y el contexto en el que actúa.

La forma de conducir una escuela adquiere rasgos particulares dependiendo de quién la dirija, "un modo particular e idiosincrático" (Fernández, 2001; Gómez-Nashiki, 2010), porque detrás de las decisiones del director están sus concepciones, creencias, valores y los principios que considera que deben guiar su tarea; lo que podríamos señalar, parafraseando a Cossío Villegas (1974), como El estilo personal de dirigir. Por esta razón, se buscó 
conocer tanto las condiciones como los resultados; es decir, cómo el director concibe y utiliza un "conjunto de reglas, estrategias y modalidades para enfrentar y resolver dificultades y tratar con tensiones y ansiedades" (Fernández, 2001), para tomar las decisiones en torno a los problemas de violencia escolar.

\section{Gestión institucional}

Está relacionada con el proceso de ejecución y desarrollo de las distintas actividades que tienen lugar en los ámbitos educativo, financiero, administrativo y técnico-pedagógico (Elizondo, 2001). Está signada por una orientación más guiada por la acción y la resolución creativa de los aspectos que surgen durante el proceso cotidiano (Alvariño et al., 2000; Elizondo, 2001; Gutiérrez-Vázquez, 2004; Zavalza, 2011). El desarrollo de la gestión se asocia a un resultado no previsible de algo que se está estructurando sobre la marcha y que trata, en la medida de lo posible, de cuidar que todos los aspectos avancen en la misma dirección. Sin embargo, en el cumplimiento de sus actividades se ve confrontado con muchos problemas, demandas, dilemas y situaciones que hacen que esta actividad se divida entre lo instituido y lo instituyente, cuyas consecuencias son inciertas y forman parte de la cotidianidad de su actividad como director.

\section{Violencia escolar}

La violencia escolar la conceptualizamos a partir de tres dimensiones (Gómez-Nashiki, Zurita y López, 2013): (1) como un recurso de poder establecido por el director para hacer valer su autoridad y mantener el control en el aula o la escuela; (2) la que se presenta entre alumnos, como parte de una fuerza abierta u oculta, con el fin de obtener algo de un individuo o de un grupo que no quiere consentir libremente; y (3) la que podemos identificar como una práctica emprendida por la propia institución educativa con el objetivo de preservar, mediante normas establecidas formal e informalmente, el orden, el control y la disciplina.

En los aspectos citados, el común denominador es que la violencia forma parte de un proceso mediante el cual un individuo o grupo violan la integridad física, social o psicológica de otra persona o grupo. La violencia inhibe el desarrollo de los alumnos, anula su potencial y puede dejar secuelas permanentes en la personalidad, pues su desarrollo se relaciona no solo con las actitudes que se le inculcan, sino con la realización estas, así como con las frustraciones sufridas en la escuela y con sus condicionamientos para favorecer o entorpecer su realización posterior (Gómez-Nashiki, 2005). 


\section{Poder y control}

El director posee varios recursos de poder para influir sobre las decisiones que se presentan cotidianamente: "Tener un puesto dentro de estas instituciones, o tenerlas bajo control, le [sic] otorga a determinadas personas una mayor capacidad para imponer al mundo su voluntad, a expensas de que otros carezcan de acceso a tales recursos de poder institucionalizados" (Goodin, 2003, p. 31). Es decir, se establece una relación de poder en la que se verifica la presencia de un sujeto activo que instrumenta la voluntad de otro pasivo, en virtud del cual el primero manda y el segundo obedece. Sin embargo, cuando los sujetos trabados en conflicto se ven impedidos de instrumentarse voluntariamente como lo exige la relación de poder, se ven obligados a ejercitar, recíprocamente, las amenazas y contraamenazas respectivas (Escobar, 1988), con lo cual da lugar a una serie de conflictos en el espacio institucional, por tanto, el control es un recurso de poder que puede adoptar diferentes tipos y modalidades. El liderazgo impuesto que asume el director indica que debe poseer múltiples "conocimientos, habilidades y actitudes que debe desarrollar para Ilevar a cabo la gestión" (Secretaría de Educación Pública, 2015), en donde el común denominador es el ejercicio del control en distintos ámbitos: administrativo, educativo, político, económico, de todos los integrantes de la institución. El control es uno de los principios básicos para lograr la estabilidad en la institución, de ahí la importancia de preservarlo.

\section{Metodología}

Los testimonios en torno a la violencia escolar se recuperaron a través de entrevistas a profundidad semiestructuradas, planteadas como un diálogo, a partir de un guion. Los temas que se trataron fueron (1) aspectos de la trayectoria docente y su experiencia como director: formación, antigüedad, especialidad; (2) las actividades de gestión que desarrollan cotidianamente con los alumnos, docentes, supervisores y padres de familia; (3) casos específicos de violencia escolar; y (4) las acciones, estrategias y soluciones que tomaron para resolverla.

De acuerdo con la perspectiva cualitativa de esta investigación, no interesó la representatividad. El tipo de muestra para esta investigación fue no probabilística, porque se buscó información profunda y detallada, y no cantidad ni estandarización. La elección de los sujetos no dependió de que tuviesen la misma probabilidad de ser elegidos, sino que obedeció a características propias de la investigación (Hernández, Fernández y Baptista, 2006), es decir, su experiencia como directores ante conflictos de violencia escolar (véase la tabla 1). 


\begin{tabular}{|c|c|}
\hline \multicolumn{2}{|c|}{ Tabla 1. Directores por institución y sexo } \\
\hline Directores & Escuelas primarias públicas \\
\hline Hombres & 5 \\
\hline Mujeres & 8 \\
\hline Total & 13 \\
\hline
\end{tabular}

Fuente: elaboración propia.

El protocolo de la entrevista consistió en explicar los objetivos del proyecto de investigación a los directores y solicitar su autorización para grabar sus testimonios y posteriormente publicarlos. Se les informó también que sus nombres no aparecerían en el trabajo, pues lo que importaba era el hecho y la narrativa de los acontecimientos. En todos los casos, los directores aceptaron sin poner restricción alguna, estuvieron de acuerdo y se mostraron dispuestos a colaborar en todo momento.

Las entrevistas fueron transcritas en su totalidad y sometidas a un proceso de análisis que consistió en lo siguiente: se diseñó un mapa conceptual para cada una, se señalaron con distintos colores las frases o palabras clave identificadas en la narrativa de los docentes y que se vinculaban con el objeto de la investigación. A partir del mapa conceptual de cada entrevista, se diseñó una matriz de las precategorías con mayor frecuencia en el discurso de los docentes -los colores que más predominaban-, donde, a partir de la narración del docente, se contrastaron los diferentes puntos de vista, es decir, se tomaron sucesivamente diferentes segmentos de información para ver con qué categorías se podían relacionar (Hammersley y Atkinson, 1994). El siguiente paso fue la triangulación, que consistió en comparar entre sí los relatos de los diferentes participantes, y posteriormente con los conceptos revisados en la teoría vinculados al objeto de investigación. De esta manera, se obtuvieron las categorías definitivas que se desarrollaron a lo largo de la investigación (Hammersley y Atkinson, 1994) (véase la tabla 2).

Tabla 2. Directores por ubicación, sexo, edad, años de servicio, años como director y grado académico

\begin{tabular}{|l|c|c|c|c|c|c|c|c|c|c|}
\hline & \multicolumn{2}{|c|}{ Sexo } & \multicolumn{2}{|c|}{ Edad } & \multicolumn{2}{c|}{$\begin{array}{c}\text { Años de } \\
\text { servicio }\end{array}$} & \multicolumn{2}{c|}{$\begin{array}{c}\text { Años } \\
\text { como } \\
\text { director }\end{array}$} & \multicolumn{2}{c|}{ Grado académico } \\
\hline & $\mathrm{H}$ & $\mathrm{M}$ & $\mathrm{H}$ & $\mathrm{M}$ & $\mathrm{H}$ & $\mathrm{M}$ & $\mathrm{H}$ & $\mathrm{M}$ & $\mathrm{H}$ & $\mathrm{M}$ \\
\hline Colima & & & & & & & & & & \\
\hline Caso n.01 & & $\times$ & & 33 & & 7 & & 3 & & Licenciatura \\
\hline Caso n. 2 & $\times$ & & 40 & & 12 & & 4 & & Licenciatura & \\
\hline Caso n. 3 & & $\times$ & & 42 & & 17 & & 6 & & Licenciatura \\
\hline Comala & & & & & & & & & & \\
\hline Caso n. 4 & & $\times$ & & 45 & & 22 & & 10 & & Maestría \\
\hline Caso n.5 & & $\times$ & & 28 & & 2 & & 1 & & Licenciatura \\
\hline
\end{tabular}




\begin{tabular}{|c|c|c|c|c|c|c|c|c|c|c|}
\hline Manzanillo & \multicolumn{2}{|c|}{ Sexo } & \multicolumn{2}{|c|}{ Edad } & \multicolumn{2}{|c|}{$\begin{array}{l}\text { Años de } \\
\text { servicio }\end{array}$} & \multicolumn{2}{|c|}{$\begin{array}{l}\text { Años } \\
\text { como } \\
\text { director }\end{array}$} & \multicolumn{2}{|c|}{ Grado académico } \\
\hline Caso n. ${ }^{\circ}$ & $x$ & & 26 & & 4 & & 1 & & Licenciatura & \\
\hline Caso n. ${ }^{\circ} 7$ & & $x$ & & 39 & & 5 & & 1 & & Licenciatura \\
\hline Caso n. ${ }^{\circ} 8$ & $x$ & & 47 & & & 13 & 5 & & Licenciatura & \\
\hline Caso n. ${ }^{9}$ & & $x$ & & 33 & & 11 & & 4 & & Licenciatura \\
\hline Tecomán & & & & & & & & & & \\
\hline Caso n. ${ }^{\circ} 10$ & & $x$ & & 44 & & 18 & & 9 & & Licenciatura \\
\hline Caso n. ${ }^{11}$ & $x$ & & 49 & & & 19 & 8 & & Licenciatura & \\
\hline $\begin{array}{l}\text { Villa de } \\
\text { Álvarez }\end{array}$ & & & & & & & & & & \\
\hline Caso n. ${ }^{12}$ & & $x$ & & 38 & & 6 & & 2 & & Licenciatura \\
\hline Cason. ${ }^{\circ} 13$ & $x$ & & 42 & & & 14 & 9 & & Licenciatura & \\
\hline
\end{tabular}

Fuente: elaboración propia.

\section{Hallazgos}

Si bien el tema de la violencia está presente en algunas de las interacciones que cotidianamente se registran entre los distintos sujetos de la institución, los testimonios de los directores entrevistados se refieren a problemas de violencia escolar cuya situación lo trataron personalmente, es decir, conflictos que no resolvieron los docentes en el aula y que Ilegaron a la dirección del plantel.

\section{Dirección e incertidumbre}

De acuerdo con la función que desempeña el director, se espera de él, y así se lo dicen: "que sepa qué hacer", cómo actuar ante cualquier eventualidad que se presente; una responsabilidad de la que están conscientes, aunque manifiestan que lo hacen "bajo mucha presión" y, en ocasiones, sin saber en un primer momento "cómo hacerle". Por ejemplo, señala una directora lo siguiente:

Directora, 42 años:

A veces, me voy a casa con muchas preocupaciones [...], pero en medio de eso hay que ir a recoger al niño [...] que preparar la comida, y así, los quehaceres de una como mujer [...], pero te cuento que hasta sueño con los problemas de la escuela [...], y es que es mucha la responsabilidad, iy es que todos esperan mucho de ti!, ¡de que a todo le hagas! [sic] [...], esto no se acaba cuando te vas a casa ¡no qué!, ¡eso ya me quedó bien claro!, es un puesto de 24 horas, (Ent. n. ${ }^{\circ}$ 8, 30.09.15). 
En otro testimonio, se manifiesta la incertidumbre que tiene la jornada cotidiana:

Directora, 26 años:

Una sale de la casa temprano y no sabes lo que te va a pasar, ¿qué te vas a encontrar en el trabajo? [...] salen los alumnos de la escuela y esto no se acaba [...], lo peor es cuando te toca un problema de violencia [...], una alumna subió al facebook una foto enseñando sus pechos sin ropa y unas de sus amigas (hace señas con las manos como si pusiera comillas) ¡se la pasaron a media escuela! ¡Fue un problemón! [...], sus papás acá querían comerse a las supuestas amigas, fue una cosa terrible, muy triste $[. .$.$] , para esto una no está preparada, ni te imaginas$ ¿cómo hacerle?, ¿cómo le haces? pero le tienes que entrar [...], para eso eres la directora ¿no? (Ent. n. ${ }^{\circ} 5$, 06.09.15).

Situaciones imprevistas que trastocan la gestión de la escuela y ante las cuales el director manifiesta su preocupación sobre cómo atender de la mejor manera el problema. La gestión bajo estas características presenta un fuerte componente de incertidumbre ante lo que el director debe enfrentar, esté preparado o no, y es lo que esperan buena parte de los integrantes de la institución.

\section{Liderazgo}

Los directores están cotidianamente puestos en el centro de atención de la escuela, y se espera que ejerzan un liderazgo de manera constante y ante cualquier circunstancia. Por esta razón, una parte fundamental de su gestión recae en el tipo de tratamiento que les da a los problemas, así como la solución que implementa, pues sus decisiones se traducirán en aspectos a favor o en señalamientos negativos a su imagen, así como a la legitimidad de su liderazgo en la institución. Al respecto se pueden mencionar los siguientes testimonios:

Directora, 45 años:

Ser directora es como ser cohetero, si dices algo: malo, si no haces nada: malo [...], ipero eso sí!, ¡todo mundo está al pendiente! [...], sabes que tienes que estar al servicio de la escuela [...], tener ganas de trabajar con todos, aunque no todos tengan ganas de trabajar contigo [...], puedes como pedir consejos, pero nadie te va a ayudar a resolver los problemas [...], le tienes que entrar, ¡eso es ser directora!, y ¡no vas a darle gusto a todos! (Ent. n. ${ }^{\circ}$, 04.10.15). 
Director, 47 años:

Cuando se han presentado problemas de violencia como peleas entre alumnos, o de que los padres se quejan de un maestro, trato de investigar bien primero [...], porque todo esto es una ;reverenda chinga!, y no es exageración, no lo arreglas bien y te anda costando hasta el trabajo, en serio [...], me dicen mis maestros que tengo mucho poder y ¿cuál? es como andar siempre al filo de la navaja [...] (Ent. n. $\left.{ }^{\circ} 11,20.10 .15\right)$.

Directora, 38 años:

Estar al frente de 100 o 150 gentes, entre niños y maestros no es fácil. Yo lo siento como mi responsabilidad de que no les pase nada, pero por lógica ¿no?, tanto niña y niño juntos pues pasan cosas, problemas $[\ldots]$, ipero hay veces que tienes que ponerte bien lista y firme! [...], como la niña que pagaba a dos de sus compañeros ipara que le pegaran a otra!, ¿puedes creer eso?, eso si ya es muy bajo ¿pues qué estamos en la calle para hacer esas cosas?, ya son como cosas de delincuentes, de mafiosos [...] (Ent. n. $\left.{ }^{\circ} 2,23.08 .15\right)$.

En los directores se advierte una preocupación constante de querer controlarlo todo, de estar informado en detalle acerca de cualquier asunto que surja en la escuela como una estrategia para mejorar la gestión. Sin embargo, la misma dinámica de la escuela presenta diversas situaciones que están fuera del control, incluso de los docentes, como son los espacios intersticiales, en donde se da un funcionamiento o conductas atípicas, como apunta Roussillon: "al lado de la institución estructurada, se organizan funcionamientos institucionales atípicos" (1989, p. 190).

\section{Control}

El control de las acciones en la institución es el común denominador de las preocupaciones que manifiestan los entrevistados, además de señalar que cuando se trata de violencia escolar "les quita mucho tiempo" y les impide llevar a cabo otras actividades. Los conflictos asociados a la violencia son importantes, en la medida en que rompen con el orden establecido y pueden detonar otros conflictos latentes, porque no se dan a nivel de un individuo, sino que involucran a otros sujetos. Dos testimonios son muy claros al respecto.

Directora, 33 años:

Uno no quiere broncas, y quiere que todo marche bien -ajá-, pero no se puede, porque no depende de ti, yo no puedo evitar que los alumnos 
se peleen, o que se porten bien, uno ¡qué más quisiera! ¿verdad? Pero no $[\ldots]$, y cuidado y no pares la bronca luego, porque crece y se va metiendo más y más gente, y ya luego no sabes cómo pararla [...] (Ent. n. $\left.{ }^{\circ} 12,27.10 .15\right)$.

El otro testimonio, señala lo complicado que es manejar este tipo de asuntos:

Directora, 42 años:

... un niño trae un cuchillo y janda amenazando a sus compañeros!, lo primero es que te espantas ¿no?, eso ya es grave, y a eso todo lo que te quita de tiempo, entre que hablas con el alumno, con la maestra, con el grupo, con el supervisor, con los padres, o sea que no tienes otro camino que invertirle tiempo y mucho, y luego échale lo que tenemos diario que hacer en la escuela [...], y eso si no hay otro problema [...], o te agarra a principio del año, no nomás se trata de sacar al alumno de la escuela iy ya!, [...] (Ent. n. $\left.{ }^{\circ} 3,03.09 .15\right)$.

La complejidad en el manejo de estos temas se torna más difícil de atender debido a la fuerte carga administrativa que tienen que cumplir y que se acentúa en coyunturas específicas. Sin embargo, cuando una situación de violencia se presenta, el director está obligado a indagar lo acontecido, por lo que llegar a una determinación implica una investigación, a través de un proceso que involucra citatorios y entrevistas con maestros, padres de familia y autoridades educativas. Desde luego que, de acuerdo con la gravedad del hecho, por ejemplo, un alumno herido, un robo en el plantel, acoso, conlleva la participación de varias instancias: Secretaría de Educación, Procuraduría General de la República, Comisión Nacional de Derechos Humanos, entre otras, lo que se traduce en mayor tiempo y una carga extra de trabajo para poder solucionarlo.

\section{Violencia}

Como lo señalan algunos entrevistados, los problemas de violencia escolar "son un dolor de cabeza", por la cantidad de implicaciones que tienen, ya sea a nivel personal, con los sujetos involucrados, lo administrativo, lo legal y lo educativo. $Y$ esto es así porque con rapidez se convierte en un tema que afecta el clima escolar y se vuelve motivo de curiosidad, intranquilidad y preocupación para los distintos integrantes de la institución, como evidencian los siguientes testimonios.

Director, 49 años:

Aquí en la escuela tenemos un problema grueso, cabrón [...], un niño le vale madre todo $[\ldots]$, muerde, pega, escupe, grita $[\ldots]$, a sus 
compañeros ¡hasta los amenaza de muerte! [...] la escuela ya toda se enteró y muchos padres de otros grupos me dijeron ¿qué vamos a hacer $[\ldots]$, ¿no sabemos por dónde entrarle?, y la supervisora me dijo que con cuidado hay que manejar este asunto, que hay que llenar los formularios, pero digo yo, ¿solucionan algo?, ipues no! [...], y el niño ¡tiene seis años! ¡Y tiene de cabeza a la escuela!, imagínate si apenas está empezando la escuela [...], los papás y otros alumnos ya lo miran con miedo. (Ent. n. ${ }^{\circ} 1,18.08 .15$ ).

Directora, 39 años:

Cuando se trata de problemas donde ya hay golpes y acusaciones la cosa cambia, los protocolos de la violencia que hay, y los formularios que hay ahora tienen sus "pros", pero muchos "contras", porque se trata de un problema que requiere de hacer cosas en el momento; son problemas que tienes que atender rápido [...], pero no sabes cómo [...], son un dolor de cabeza porque a final de cuentas no puedes resolver con formatos solamente, pero ¡cuidadito eh! iy no llenes los formatos, porque te metes en un problema grave! (Ent. n. ${ }^{\circ}$ 5, 06.09.15).

Director, 40 años:

Lo que he aprendido es que cada problema es diferente, iy donde hay golpes pues más!, o cuando hay alumnos maltratados, porque eso es ya cosa de agresiones, de que hubo daños, robos [...], y eso provoca mucha inquietud en los alumnos, en los profesores, en la escuela, porque para lo malo ya sabes: luego, luego se sabe [...], pero esos son los problemas que te caen y órale te las ingenias iporque esto no viene en el librito! (risas) (Ent. n. $\left.{ }^{\circ} 2,23.08 .15\right)$.

Los testimonios muestran la dificultad para hacer frente a este tipo de problemas, tanto por lo administrativo con que se tiene que reportar, como por el cuidado para poder tomar una decisión. Es importante mencionar que si bien los protocolos registran actos de violencia, se recomiendan como una primera medida; los entrevistados advierten ciertas limitantes en su aplicación, y muestran que hace falta complementarlo con mayor capacitación en temas como manejo de conflictos, estrategias de convivencia pacífica, por ejemplo.

\section{Implicaciones para la gestión}

El tema del conflicto es un reto; más aún si este deriva en violencia, porque de acuerdo con los entrevistados, se trata de un asunto complejo con múltiples implicaciones y consecuencias, que obliga a manejarlo con 
muchas instancias, y en donde la figura del director es de negociador e investigador, porque ya "no se trata de señalar quién tuvo la culpa o poner un castigo, eso era antes". Así lo dice una de las entrevistadas:

Directora, 39 años:

Siempre he dicho que ser directora es como ser todóloga, y con muchas ganas de entrarle a lo que se presente, ¡de veras; [...] vives al día, no digo del papeleo, de las reuniones y de los reportes, porque eso ya al primer año te lo aprendes [...], lo digo cuando hay problemas [...], de violencia, de burlas, del bullying [...], y ya no se trata de señalar quién tuvo la culpa o poner un castigo, eso era antes [...], es algo latoso porque hay que hablar con los padres, decirles a las autoridades, o sea a todo mundo [...], y todos te presionan [...] y todos dicen que tienen la razón, y pones la solución y siempre alguien se queja, nomás no quedas bien nunca. (Ent. n. ${ }^{\circ} 3,13.09 .15$ ).

En relación con las habilidades que debe poseer un director, los entrevistados reconocen que es necesario "echar mano de la experiencia", "ser cuidadoso", "pensar las cosas bien antes de hacer algo", "no ser impulsivo", "ponerte listo", ente otras expresiones que denotan el cuidado para poder tomar una decisión.

\section{Normatividad}

Con la inclusión de nuevas disposiciones normativas, por ejemplo los protocolos, para atender los problemas de la violencia escolar, los directivos manifiestan posiciones encontradas- Sin embargo, coinciden en que el problema de fondo es la falta de capacitación en todos los integrantes de la escuela, incluyendo a los padres de familia, ante este tipo de situaciones. Al respecto dos puntos de vista reflejan muy bien estos aspectos:

Director, 38 años:

Si los problemas que hay en las escuelas se pudieran solucionar, o ya digamos bajar con las leyes o reglamentos ya no tendríamos problemas desde hace mucho, ipero no! Aquí en las escuelas la cosa es diferente [...], pero lo latoso es Ilenar todo lo que piden, esto sí es un martirio, es más burocracia [...], luego ya no sabes qué es peor, si el problema o el llenado de los formatos [...] (Ent. n. $\left.{ }^{\circ} 8,30.09 .15\right)$.

Directora, 44 años:

Cuando nos informaron de que tenía que llenar más formatos en problemas de violencia [...] la verdad, me dije: -¡más trabajo! [...], además de oficios, ¡los benditos formatos! [...], y en la dirección ¡hay 
tantas cosas qué hacer! [...], yo creo que lo que nos hacen falta son herramientas, a mí y a mi personal, digo en cosas de: ¿cómo hablarles a los padres?, ¿cómo hablar con un niño que pega?, ¿qué decirles a sus compañeros?, ¿cuáles son los límites que hay que poner? [...], de eso no sabemos mucho (Ent. n. $\left.{ }^{\circ} 6,12.09 .15\right)$.

Las opiniones apuntan a que el proceso burocrático que representan los protocolos es poco ágil para atender los problemas de violencia escolar, y no los consideran como herramientas de ayuda.

En cuanto a los Consejos Escolares de Participación Social se han establecido distintos comités, entre ellos el de Desaliento de las Prácticas que Generen Violencia entre Pares. Sin embargo, de acuerdo con los directores, a los docentes no les Ilama mucho la atención, porque todos quieren estar en los comités de Lectura y Nuevas tecnologías. Así lo mencionan las siguientes entrevistas:

Directora, 33 años:

Cuando en el Consejo de Participación Social se habla de quién le entra al comité nadie quiere y a la hora de ponerlos en marcha no hay interés [...], a los padres y maestros les Ilama más la atención que sus hijos lean [...], les preocupa mucho más la lectura o lo de las tecnologías [...], en esos comités todos se quieren apuntar, y creo que es porque los maestros le saben más a eso que a lo de la convivencia ¿no? (Ent. n. ${ }^{\circ}$ 9, 04.10.15).

Otro testimonio similar es el siguiente:

Director, 42 años:

Cuando en el Consejo discutimos quién se apunta a los Comités nadie quiere el de convivencia o el de violencia, son de los que menos escogen, si hay responsables, pero no es como lo que quieren ¿verdad? (Ent. n. ${ }^{\circ}$ 2, 23.08.15).

La violencia afecta el clima escolar y las relaciones entre los integrantes de la institución, por lo que se convierte en un reto importante; no obstante, los directores identifican la toma de decisiones y sus repercusiones como un proceso que poco se valora y que cuenta con escaso apoyo por parte de su personal. En este sentido, el señalamiento de los directores apunta a que estos procesos no pueden darse de manera aislada, sino que deben involucrarse los distintos sujetos, con la finalidad de que se apoyen colectivamente las decisiones. 


\section{Los padres de familia}

En relación con los padres de familia, los directores identifican dos posiciones: por una parte, están los que aceptan los señalamientos que hacen de sus hijos, ya sea como víctimas o agresores, y por otra, los padres que no aceptan lo que el director señala y rechazan a priori cualquier señalamiento. En ambos casos, coinciden en el poco apoyo que se brinda a los estudiantes desde sus respectivos hogares. Así lo manifiestan dos directores:

Directora, 49 años:

Los papás no ayudan [...], creen que uno tiene que hacer su trabajo, de todo se quejan siempre, pero no ayudan, o muy poco [...], han venido aquí desde los que a todo te dicen que sí y no hacen nada, pero eso sí te lo juran que le van a echar ganas para que su hijo se porte bien y nada ¿verdad? [...] (Ent. n. $\left.{ }^{\circ} .13,28,10.15\right)$.

Director, 40 años:

Hay padres que no aceptan nada, les dices algo de su hijo y se ponen al brinco y no los haces entender [...], les demuestras, les enseñas lo que hace su hijo y nada [...], hay casos en que no aceptan que su hijo se porta mal, y ahí ¿qué?, ¿qué haces?, y no hacen nada, le dejan a la escuela todo el paquetote, y ya me ves ahí con la supervisora, con los de la secretaría, con los del sindicato, es difícil, muy difícil, no te creas. (Ent. n. $\left.{ }^{\circ} 1,18.08 .15\right)$.

El tipo de participación que demandan los directores de los padres de familia se centra en el apoyo a sus decisiones y en aspectos relacionados con el comportamiento de su hijo en relación con el cumplimiento de las normas y reglas de la escuela, lo cual está sujeto a varios factores, por ejemplo, el desinterés por parte de los padres en la educación de sus hijos o en el desencuentro de opiniones que mantengan con el director.

\section{Discusión}

El análisis institucional permitió identificar cómo las acciones de maestros, padres de familia, alumnos, supervisor y el director definen y redefinen la existencia y las características que adopta la institución en un momento determinado, y son confrontadas cotidianamente con las condiciones objetivas de existencia. 
Se consideraron dos vertientes en el análisis: por una parte, la caracterización del rol del director, a través de lo que señalan y establecen las normas en los reglamentos, en la perspectiva del deber ser, y por otro lado, las acciones y decisiones que adoptaron, percibieron y modificaron de acuerdo a sus intereses, valores y formas de valorar las distintas situaciones y dinámicas ante un hecho determinado de violencia escolar, es decir, el ser. Una confrontación entre lo instituido y lo instituyente de las distintas funciones directivas en cuanto a los problemas de violencia escolar muestra que: "... lo relevante no es lo que las personas se supone que deben hacer, sino lo que en realidad hacen" (Goodin 2003, p. 27).

En torno a la figura del director se despliega toda una serie de situaciones de distinta índole en el espacio de la escuela y de los hogares de los alumnos, pues como apunta Pastrana (1996), la escuela "adopta" a los alumnos y también a los padres; incluso, algunas madres de familia se refieren a los directores como los "jefes de la escuela", en reconocimiento a su lugar y las funciones de liderazgo que desempeñan.

Los entrevistados señalan que llevan a cabo su trabajo en medio de muchas presiones, tanto las que se generan a diario de manera rutinaria (planeación, informes, reportes, reuniones, etcétera) como las imprevistas, por ejemplo los conflictos cotidianos entre los distintos sujetos que requieren de respuesta inmediata. Es decir, se encuentran en tensión entre diversos asuntos por resolver, así como en la búsqueda de apoyo por parte de su personal. Sin embargo, enfatizan que se trata de un puesto "en el que con nadie se queda bien", además de sentir que su labor al frente de la institución no recibe suficiente reconocimiento.

Las decisiones del director están mediadas por un cúmulo de intereses, puntos de vista y presiones de parte de los sujetos; sin embargo, está obligado a decidir a corto plazo, lo que se traduce en una presión más. De acuerdo con los entrevistados, en torno a las decisiones que toma el director sobre diversos aspectos y problemas, se cuida que estas sean las más adecuadas a las circunstancias, y lo hace a partir de su experiencia, así como de la interpretación de la norma, el contexto y las circunstancias del evento, pero también, a partir de las habilidades personales que posee o que va desarrollando a lo largo de su trayectoria para dar solución al problema. Es importante mencionar que, según los testimonios recabados, los directores no reciben una preparación específica de inducción al puesto, sino que se van formando sobre la marcha, en el quehacer cotidiano del desarrollo de la gestión; por esta razón, el insistente llamado a recibir una capacitación para todo el personal para que pueda desempeñar las funciones directivas adecuadamente. 
En la escuela se registran distintos conflictos y formas de violencia escolar, por lo que cuando un conflicto llega a la dirección es signo de que el problema está más allá de las habilidades del docente; en consecuencia, el director tiene que intervenir, de ahí la insistencia de los entrevistados en que los docentes cuenten con capacitación para resolver en las aulas los distintos problemas que surgen.

La autonomía de las decisiones del director en temas de violencia está sujeta a normas e instrumentos como son los protocolos. No obstante, tanto los directores jóvenes como los que ya tienen mayor antigüedad coinciden en que para poder decidir con más elementos es necesario estar mejor capacitados.

El común denominador de los testimonios señala, por una parte, que lo más relevante es mantener "todo bajo control", y en segundo lugar, resolver los conflictos rápidamente. Sin embargo, se aprecian dos posturas al respecto: algunos directores consideran que los problemas de violencia escolar son una amenaza que hay que solucionar de manera rápida y de raíz, además de insistir en que lo que no se ajuste a la norma debe sancionarse; una suerte de estilo rígido de dirección. En contraste, otro grupo de directores afronta estos problemas de manera diferente, intentando llegar a soluciones mediante la negociación, y utilizan los instrumentos legales -los protocolos y reglamentos- como guías de acción para resolver los conflictos, es decir, un estilo más enfocado a la negociación con las partes para resolver el problema.

Ante decisiones apremiantes relacionadas con situaciones de violencia (física, verbal, psicológica o sexuada), los directores consideran que es necesario difundir los protocolos entre padres, maestros y alumnos, pues si bien estos instrumentos prescriben qué hacer, hace falta comprometerse con la filosofía que establecen, aplicar sus indicaciones y adecuarlos a cada contexto, con la finalidad de que sean útiles, pues de otra manera se corre el riesgo de que se conviertan en instrumentos sin fines prácticos, o que solo ayuden a contabilizar los hechos violentos, como lo han señalado los directores.

En lo que se refiere a la violencia escolar coinciden en que se trata de un tema difícil de manejar y solucionar, porque implica mucha investigación de los hechos, una importante inversión de tiempo para poder tomar una decisión, lo que se suma a las tareas en la dirección que, por lo regular, siempre tiene muchas actividades que atender. Además, ponen de relieve que sus decisiones son siempre cuestionadas y sujetas a debate, al señalar por ejemplo que: "a nadie le das gusto".

Los directores señalan que han recibido cierta información y capacitación acerca de las nuevas disposiciones para mejorar la convivencia, así como para atender los problemas de violencia escolar. Sin embargo, 
coinciden en que es necesario implementar una capacitación integral, contextualizada y permanente, dirigida a docentes, alumnos, supervisores, y desde luego a ellos, con la finalidad de contar con las herramientas suficientes para atender estos asuntos, pues casi siempre son esfuerzos aislados que no tienen continuidad, por lo que es importante considerar espacios de asesoría y seguimiento permanente en la escuela, que estén "más allá de modas", como señaló uno de los entrevistados.

El tema de la violencia está muy ligado a los hogares de los alumnos, pues son los padres que acuden al Ilamado de los docentes y directores para resolver los distintos problemas que presentan sus hijos, ya sea como agresores o como víctimas, y es un reto negociar con ellos, pues parten de opiniones y juicios parciales acerca del funcionamiento de la escuela y de la conducta de su hijo, producto del desconocimiento de la dinámica cotidiana, por lo que recomiendan mayor participación de los padres en las distintas actividades de la escuela.

\section{Referencias}

Abdolhamid A. y Mehdinezhad, V. (2015). The relationship between the school principals' collaborative leadership style and teachers' self-efficacy Palestrica of the third millennium. Civilization and Sport, 16(2), 125-131. Recuperado de http://bvirtual.ucol.mx/url. php? u=http 3A $\sim 2$ F 2Fsearch.ebscohost.com 2Flogin.aspx 3Fauthtype 3Dip 2Cuid 26profile 3Dehost 26defaultdb 3Da9h.

Alvariño, C. et al. (2000). Gestión escolar. Un estado del arte de la literatura. Paideia, 29, 15-43.

Ball, S. J. (1989). La micropolítica de la escuela. Hacia una teoría de la organización escolar. Barcelona: Paidós-MEC.

Berguer, T. y Luckmann, N. (1997). La construcción social de la realidad. Buenos Aires: Amorrortu.

Carriego, C. (2005). Los desafíos de la gestión escolar. Una investigación cualitativa. La Crujía: Argentina. Stella.

Cossio Villegas, D. (1974) El estilo personal de gobernar. Cuadernos Joaquín Mortiz: México.

Elizondo, A. (2001). (Coord.). La nueva escuela. Dirección, liderazgo y gestión escolar. Paidós: México.

Enriquez, E. (2005). La institución y las organizaciones en la educación y la formación. Argentina: Ediciones Novedades Educativas, Universidad de Buenos Aires.

Escobar, M. (1988). Contribución al estudio del poder. Acatlán, México: UNAM-ENEP. 
Ezpeleta, J. y Furlan, A. (1992). (Comp.). La gestión pedagógica de la escuela. México: Correo de la Unesco

Fernández, L. (1995). Instituciones educativas. Dinámicas institucionales en situaciones críticas. Buenos Aires: Paidós.

García, J. M.; Slater, Ch. y López, G. (2010). El director novel de primaria. Problemas que enfrenta en su primer año. Revista Mexicana de Investigación Educativa. 15(47), 1051-1073.

Gómez-Nashiki A. (2005). Violencia e institución educativa. Revista Mexicana de Investigación Educativa, 10(26), 693-718.

Gómez-Nashiki A. (2010). Micropolítica escolar y procesos de cambio. El papel del supervisor en una institución educativa. Revista Mexicana de Investigación Educativa. 15(46), 771-802.

Gómez-Nashiki, A.; Zurita, Ú. y López, S. (2013). La violencia escolar en México. México: Cal y Arena, Sistema Nacional de Educación a Distancia, Universidad de Colima.

Goodin, R. (2003). (Comp.). Teoría del diseño institucional. Madrid: Gedisa.

Gutiérrez-Vázquez, J. M. (2004). Con paso lento y agitadamente. Ensayos educativos. México: Die-Cinvestav.

Hammersley, M. y Atkinson, P. (1994). Etnografía. Métodos de investigación. Barcelona: Paidós.

Hernández, S.; Fernández, C. y Baptista, P. (2006). Metodología de la investigación. México: Mc Graw-Hill.

Karakose, T.; Yirci, R. y Kocabas, I. (2014). A qualitative study of the novice principals' problems in the school management process and solutions. Pakistan Journal of Statisctics, 30(6), 1365-1378.

Kaya, A. (2015). The relationship between spiritual leadership and organizational citizenship behaviors: A research on school principals' behaviors. Educational Sciences: Theory and Practice, 15(3), 597-606. DOI: 10.12738/estp.2015.3.1988.

Knight, J. (1992). Institutions and social conflict. Nueva York: Cambridge University Press.

López-Yáñez, J.; García-Jiménez, E.; Rodríguez, N.; Moreta-Jurado, B. y Bellerín, A. (2014). El liderazgo escolar a través del análisis de la actividad diaria de los directores. Revista Iberoamericana sobre Calidad, Eficacia y Cambio en Educación. 12(5), 61-78. Recuperado de http://www.rinace.net/reice/numeros/arts/vol12nu m5/art4.pdf.

Lourau, R. (1991). El análisis institucional. Buenos Aires: Amorrortu. 
Meng, J. y Berguer, B. (2012). Strategic leadership in public relations: An integrated conceptual framework. Public Relations Review, 38(2), 336-338. DOI: 10.1016/j.pubrev.2012.01.004. Recuperado de http://bvirtual.ucol.mx/url.php?u=http 3A 2F 2Fsearch.ebscohost. com $\sim$ Flogin.aspx $\sim 3$ Fauthtype 3Dip 2Cuid 26profile $\sim$ 3Dehost 26defaultdb 3Da9h.

Mintzberg, H. (1983). La naturaleza del trabajo directivo. Barcelona: Ariel. Morgade (2010). Mujeres que dirigen poniendo el cuerpo. Poder. Autoridad y género en la escuela primaria. Argentina: Noveduc.

Morris, S.; Guat. T. y Coleman, M. (1999). Leadership stereotypes and styles of female Singaporean principals compare. A Journal of Comparative Education, 29(2), 191-202.

Murillo, F. J. y Hernández-Castilla, R. (2015). Liderazgo para el aprendizaje: ¿Qué tareas de los directores y directoras escolares son las que más inciden en el aprendizaje de los estudiantes? Relieve, 21(1), 1-20. Recuperado de http://bvirtual.ucol.mx/url.php?u=http 3A 2F 2 Fsearch.ebscohost.com 2 Flogin.aspx 3 Fauthtype 3Dip 2Cuid 26profile 3Dehost 26defaultdb 3Da9h. DoI: 10.7203/relieve.21.1.5015.

Ng, S.W. y Chan, Y. F. (2014). Aspiring principals' perceptions of applicability of acquired leadership capacities in the school context. International Studies in Educational Administration Commonwealth Council for Educational Administration y Management (CCEAM), 42(3), 85-101. http://bvirtual.ucol.mx/url.php?u=http 3A 2F 2Fsearch.ebscohost. com 2Flogin.aspx 3Fauthtype 3Dip 2Cuid 26profile 3Dehost 26defaultdb 3Da9h.

Pastrana, L. (1996). Organización, dirección y gestión (tesis de maestría). DIE-Cinvestav: México.

Pereira, A. (2014). Liderazgo líquido, una propuesta para enfrentar la incertidumbre y riesgo. Pensamiento y Gestión. Universidad del Norte, 37, 97-113.

Roussillon, R. (1989). Espacios y prácticas institucionales. La liberación y el intersticio. En R. Käes et al. La institución y las instituciones. Estudios psicoanalíticos. Buenos Aires: Paidós.

Santos-Guerra, M. A. (2015). Las feromonas de la manzana El valor educativo de la dirección escolar. España: GRAÓ.

Secretaría de Educación Pública (2015). Perfil, parámetros e indicadores para personal con funciones de director y supervisor, Subsecretaría de Educación Básica y Coordinación Nacional del Servicio Profesional Docente. México. Recuperado de http://servicioprofesionaldocente. sep.gob.mx/content/ba/docs/2015/permanencia/parametros_indicadores/ppi_desempeno_directivos_supervisores.pdf. 
Tesio, A. (2008). Transformaciones institucionales de una escuela en un "lugar difícil": la construcción de la autoridad y el papel del director. Cuadernos de Educación, 6(6), 211-224.

Welzer-Lang, D. (2007). La violencia doméstica a través de 60 preguntas y 59 respuestas. Madrid: Alianza.

Wood, R. y Zalud, G. (1996). Opinions of rural Mid-Western principals toward violence in schools. Education, 116(3), 397. Recuperado de http://bvirtual.ucol.mx/url.php?u=http 3A 2F 2Fsearch.ebscohost.com 2Flogin.aspx 3Fauthtype 3Dip 2Cuid 26profile 3Dehost 26defaultdb 3Da9h.

Zavalza, M. A. (2011). Profesores y profesión docente. Entre el ser y el estar. Madrid: Narcea. 